\section{Severe asthma}

\section{M1 CONCORDANCE TO ASTHMA MEDICATIONS IN PATIENTS RECEIVING LONG-TERM OMALIZUMAB TREATMENT}

MS Elsherbiny, V Mitchell, AH Mansur, L Afridi, B Ahitan; Severe and Brittle Asthma Unit (SBAU) at Heartlands Hospital, Heart of England NHS Foundation Trust, Birmingham, United Kingdom

\subsection{6/thoraxjnl-2013-204457.411}

Introduction It is estimated that $5 \%$ of adult asthma patients have uncontrolled asthma despite high dose corticosteroid therapy. Omalizumab is a monoclonal anti-IgE antibody that has been shown to improve asthma control. ${ }^{1}$ As asthma control improves, concordance to other prescribed asthma medication may decline which may undermine omalizumab effectiveness. The extent of such non-concordance is explored in this study. Methods The records of 40 patients receiving long-term omalizumab treatment have been reviewed for concordance. Medication history was retrieved for all patients, which included all prescriptions issued by their General Practitioner over most recent twelve months, detailing the doses and the number of tablets and/or inhalers patients received. Patients were classified as non-concordant if their medication history showed less than $75 \%$ adherence. Concordant and nonconcordant groups were compared in relation to asthma control questionnaire (ACQ), Fractional exhaled nitric oxide (FeNO), forced expiratory volume in one second (FEV1), and number of asthma related admissions and frequency of severe steroid requiring asthma exacerbations at baseline line of omalizumab treatment.

Results Sufficient information was only available for 25 patients (20 female) receiving long-term omalizumab treatment with a mean age of 44 years (range 19 to 68 years) and mean weight of $78 \mathrm{~kg}(\mathrm{SD}=20.78)$. The prevalence of non-concordance was $64 \%(\mathrm{n}=16)$. All non-concordant patients had $50 \%$ or less prescription filling history. Overall non-concordant patients had a higher FEV1 before starting omalizumab treatment (mean FEV1 $=1.865,95 \%$ predicted) compared to concordant patients (mean FEV1 $=1.401,95 \%$ predicted), better ACQ scores (mean $=2.26$ ), less number of patients on oral steroids $(56.25 \% \mathrm{n}=9)$ compared to $(66.7 \% \mathrm{n}=6)$.

Conclusion The prevalence of non-concordance in patients receiving long-term omalizumab treatment is high and may undermine treatment effectiveness. The non-concordant patients had overall less severe asthma than concordant patients. Further research is needed to determine the effect of such non-concordance on the effectiveness of omalizumab in the long term.

\section{REFERENCE}

1. Efficacy and safety of a recombinant anti-immunoglobulin $E$ antibody (omalizumab) in severe allergic asthma. Holgate ST, Omalizumab 011 International Study Group.

\section{M2 BONE MINERAL DENSITY CHANGES IN PATIENTS IN THE SEVERE ASTHMA CLINIC ON ORAL CORTICOSTEROIDS}

HGT Brice, DJ Wilkinson, R Daly, G Tavernier, RM Niven; UHSM, Manchester, England

\subsection{6/thoraxjnl-2013-204457.412}

Objectives How effective are bone sparing medications (BSM)? Is bone mineral density (BMD) change related to other clinical variables?

Background Regular oral corticosteroids (OCSs) cause several side effects including BMD loss, occurring at varying rates in different patients.
Method Patients attending the specialist severe asthma clinic with two available bone densitometry scans since 2007 were identified and the hip and spine BMD change calculated. The cumulative steroid dose (pre and post first scan) and risk factors for osteoporosis were also recorded, including current bone protection.

Results Yearly hip BMD change and average cumulative steroid dose did not correlate for all patients $(n=59$ Pearson $=-0.083$ $\mathrm{p} 0.53$ ) or for patients not on BSM ( $\mathrm{n}=27$ Pearson $=-0.26$ p0.19). Yearly spine BMD change also did not correlate for all patients $(\mathrm{n}=59$ Pearson $=0.012 \mathrm{p} 0.19)$ or for patients not on BSM $(\mathrm{n}=27$ Pearson $=-0.22 \mathrm{p} 0.28)$.

Body mass index (BMI) predicted a higher $\mathrm{t}$-score on the first DXA scan; hip $(\mathrm{n}=59)$ (Pearson $=0.59 \mathrm{p}<0.001)$ and spine $(\mathrm{n}=59)($ Pearson $=0.53 \mathrm{p}<0.001)$. However between scans, although not statistically significant, a higher BMI correlated with a greater decrease in BMD.

Analysing the effect of BSM (see figure: spine BMD change) showed spine BMD improved over time for patients taking a bisphosphonate $(\mathrm{n}=59) \mathrm{p} 0.029$. However when compared to patients on calcium/vitamin D or no BSM, patients on a bisphosphonate and calcium/vitamin $\mathrm{D}$ had a greater improvement $(+1.5 \%$ average BMD change, p0.039) than patients on a bisphosphonate alone $(+1.19 \%$ average BMD change, $\mathrm{p} 0.21)$. Conclusion OCS use causes a decrease in BMD which is greater in the spine. In this small population, cumulative steroid dose did not correlate to BMD change over time. Bisphosphonate and calcium/vitamin D demonstrated benefit in spine BMD between scans, whereas the benefit of bisphosphonate alone was not statistically significant.

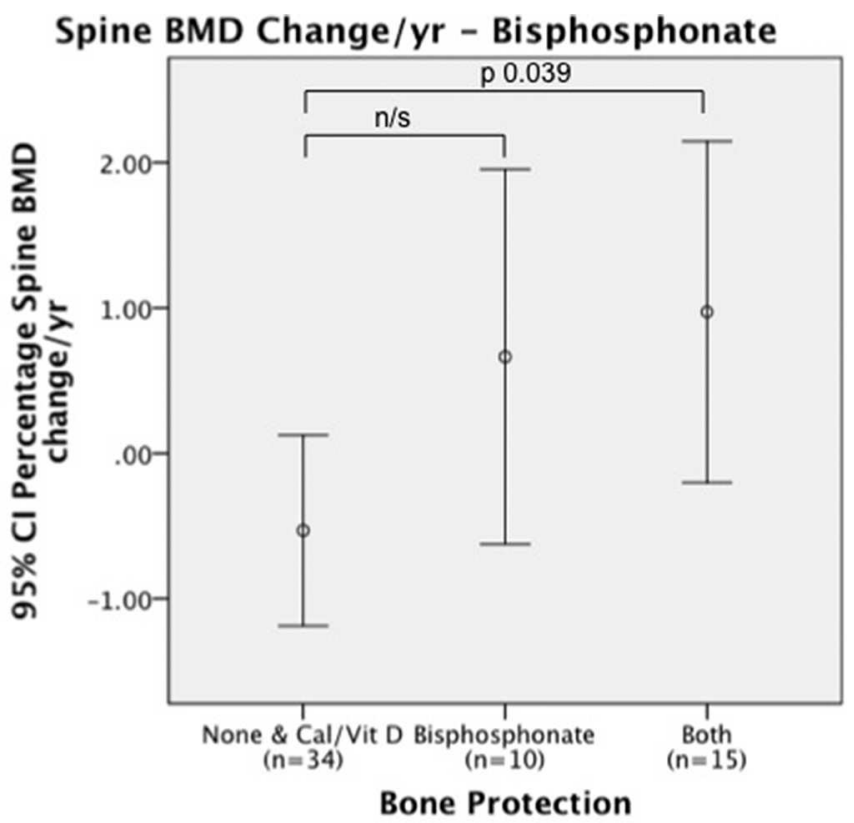

Abstract M2 Figure 1.

\section{M3 EVALUATION OF PHYSICAL ACTIVITY IN PATIENTS WITH SEVERE ASTHMA-ARE WE GETTING IT RIGHT?}

LJF Grillo, S Greenwood, J Hull, A Menzies Gow; The Royal Brompton NHS Foundation Trust, London, UK

\subsection{6/thoraxjnl-2013-204457.413}

Introduction Of the 5 million patients who receive medication for asthma approximately $5 \%$ have severe or difficult to treat 